\title{
The association of low free testosterone with histological severity of nonalcoholic fatty liver disease in Japanese men
}

\begin{abstract}
Aim: Testosterone deficiency in men is associated with an increased risk of obesity, metabolic syndrome and type 2 diabetes. However, the relationship between nonalcoholic fatty liver disease (NAFLD) and the levels of free testosterone (FT) in the serum remains unknown. Our aim was to investigate the association of serum levels of FT in male patients with NAFLD.
\end{abstract}

Methods: Serum levels of FT were measured in 66 Japanese men with NAFLD and in 82 age-matched healthy men. Liver histology was also evaluated in 44 patients with NAFLD.

Results: The serum levels of FT in male patients with NAFLD were significantly lower (median, $7.0 \mathrm{pg} / \mathrm{ml}$ ) compared with those in the control population (median, $9.1 \mathrm{pg}$ / $\mathrm{ml}, P<0.001)$. Of the 44 patients with NAFLD who received liver biopsies, 25 were diagnosed with nonalcoholic steatohepatitis (NASH). Patients with NASH showed significantly lower levels of FT (median, $6.0 \mathrm{pg} / \mathrm{ml}$ ) than those with nonalcoholic fatty liver (NAFL) (median, $8.9 \mathrm{pg} / \mathrm{ml}, P<0.001$ ). The area under the receiver operating characteristic curve for FT in patients with and without NASH was 0.832. Levels of FT decreased significantly with the increased incidence of lobular inflammation $(P<0.001)$, hepatocyte ballooning $(P<0.05)$, NAFLD activity score $(P<0.05)$, and fibrosis $(P<0.001)$. In multiple regressions, the association between FT and NASH persisted after the adjustment for age, BMI, and insulin resistance.

Conclusion: FT levels are lower in males with NAFLD compared to control population. Low levels of circulating FT are an independent predictor for the development of male $\mathrm{NASH}$, independent of age, BMI and insulin resistance.

Keywords: nonalcoholic fatty liver disease, insulin resistance, fibrosis, testosterone, nonalcoholic steatohepatitis
Volume 2 Issue 4 - 2015

Yoshio Sumida,' Michiaki Fukui, ${ }^{2}$ Jintetsu Soh, ${ }^{3}$ Saiyu Tanaka, ${ }^{4}$ Shunsuke Imai, ${ }^{5}$ Hiroshi

Mochizuki, ${ }^{6}$ Eijiro Okajima, ${ }^{6}$ Hiroyoshi

Taketani,' Tasuku Hara,' Yuya Seko,' Hiroshi Ishiba,' Akira Okajima,' Takafumi Senmaru, ${ }^{2}$ Kojiroh Mori, ${ }^{4}$ Kanji Yamaguchi,' Michihisa Moriguchi,' Hironori Mitsuyoshi,' Kazuyuki Kanemasa, ${ }^{4}$ Kohichiroh Yasui,' Masahito Minami,' 'Yoshito Itoh'

'Department of Gastroenterology and Hepatology, Kyoto Prefectural University of Medicine, Kyoto

${ }^{2}$ Department of Endocrinology and Metabolism, Kyoto Prefectural University of Medicine, Kyoto

${ }^{3}$ Department of Urology, Kyoto Prefectural University of Medicine, Kyoto

${ }^{4}$ Center for Digestive and Liver Diseases, Nara City Hospital, Japan

${ }^{5}$ Department of Pathology, Nara City Hospital, Japan

${ }^{6}$ Department of Urology, Nara City Hospital, Japan

Correspondence: Yoshio Sumida, Department of Gastroenterology and Hepatology, Kyoto Prefectural University of Medicine, Kawaramachi-Hirokoji, Kamigyo-ku, Kyoto 6028566, Japan, Tel +8I-75-25I-55I9, Fax +8I-75-25I-7I0, Emailsumida@koto.kpu-m.ac.jp

Received:June 15, 2015 | Published: August 25, 2015
Abbreviations: DHEA, dehydroepiandrosterone; FT, free testosterone; IGF-1, insulin-like growth factor-1; NAFLD, nonalcoholic fatty liver disease; NASH, nonalcoholic steatohepatitis; NAFL, nonalcoholic fatty liver

\section{Introduction}

Nonalcoholic fatty liver disease (NAFLD), a hepatic manifestation of metabolic syndrome, is the most common chronic liver disease (CLD) worldwide. NAFLD includes non alcoholic fatty liver (NAFL), nonalcoholic steatohepatitis (NASH), and NASH-related liver cirrhosis (LC) or hepatocellular carcinoma (HCC). ${ }^{1-3}$ In Japan, the current prevalence of NAFLD is estimated to be $25-30 \%$ while that of NASH is thought to be $2-3 \%$ in the general population. 4,5 The biological basis of the histological diversity in the severity of NAFLD remains unknown, although more advanced NASH is characterized by insulin resistance,${ }^{6}$ oxidative stress,${ }^{7,8}$ and hormone deficiency. ${ }^{9}$ Serum testosterone concentrations decline progressively with age in men. ${ }^{10}$ Some aging men develop a condition of suppressed serum testosterone levels, which is associated with diffuse sexual, physical and psychological symptoms. Several terms are used for this syndrome, but late-onset hypogonadism $(\mathrm{LOH})$ is preferred. $\mathrm{LOH}$ is associated with an increased risk of chronic diseases, including obesity, metabolic syndrome, diabetes, cardiovascular disease, and atherosclerosis. The Japanese Urological Association and the Japanese Association for Men's Health have recommended a diagnosis of LOH based on FT values. ${ }^{11,12}$ Low levels of serum FT are associated with metabolic syndrome, ${ }^{13}$ type 2 diabetes, ${ }^{14}$ atherosclerosis, ${ }^{15,16}$ erectile dysfunction ${ }^{17}$ and sarcopenia. ${ }^{18}$ No studies have investigated the association between FT and NAFLD, although a few reports have addressed the potential relationship between the levels of total testosterone and NAFLD. ${ }^{19,20}$ However, histological evaluation was not performed to differentiate NASH from NAFL in those studies. Therefore, we examined FT by direct immunoassay and evaluated its association with NAFLD in adult males. The aim of this study was to investigate if the endogenous androgen concentration is lower in a relatively large number of Japanese patients with NAFLD compared with healthy men and to identify what factors may be associated with low serum FT concentrations in men with NAFLD.

\section{Methods}

\section{Patients}

A total of $66 \mathrm{male}$ Japanese patients with well-characterized NAFLD ( $>18$ years) were included in this study. Diagnosis was 
confirmed by liver biopsy in 44 of the 66 patients. These were consecutively biopsied patients seen at the Center for Digestive and Liver Diseases, Nara City Hospital during the period of 2011-2013 or at the Department of Gastroenterology and Hepatology, Kyoto Prefectural University of Medicine during the period of 2012-2013. The diagnosis of NAFLD was based on the following criteria:

1. persistent elevations in transaminase activity for more than 6 months,

2. liver biopsy showing steatosis in at least $5 \%$ of hepatocytesor fatty liver by imaging studies (US/CT) and

3. Appropriate exclusion of liver diseases of other etiology including viral hepatitis, autoimmune hepatitis, drug-induced liver disease, primary biliary cirrhosis, biliary obstruction, hemochromatosis, and Wilson's disease.

Patients who consumed more than $20 \mathrm{~g}$ of alcohol per day and patients with evidence of LC or HCC were excluded from the present study. Written informed consent was obtained from all patients at the time of their liver biopsy, and the study was conducted in accordance with the Declaration of Helsinki and approved by the ethical committee of each institution. In addition, 82 age-matched healthy men who received health checkups were also enrolled as the control group. All patients in the control population presented with normal ALT levels $(<30 \mathrm{IU} / \mathrm{L})^{21}$ without evidence of fatty liver as evaluated by imaging studies. We excluded patients who received steatogenic drugs such as gluco corticoids, estrogen, amiodarone, methotrexate, and sodium valproate. Patients were excluded if they had been castrated for the treatment of testicular or prostate cancer or if they were taking any medications known to affect the concentration of sex hormones (e.g. anti androgenic agents for prostate cancer). We also excluded patients with low levels of FT secondary to pituitary adenoma or Kleinfelter syndrome. Two NAFLD patients who received antidepressant drugs were included in this analysis, although antidepressant drugs can affect serum FT levels. Self-report data on smoking habits such as number of cigarettes smoked per day and duration of smoking (measured in years) were collected during personal interviews. Participants were categorized as current smokers, past smokers, and never smokers. Men who had smoked cigarettes for 6 months or longer and reported that they were still smoking at the time of liver biopsy were considered "current smokers", whereas men who had stopped smoking since more than 6months prior to liver biopsy were considered "past smokers" and those who never smoked were "never smokers".

\section{Clinical laboratory parameters}

Venous blood samples were obtained in the morning after a 12 hour overnight fast. The laboratory evaluation in all patients included a blood cell count and the measurement of the levels of the following: aspartate aminotransferase (AST), alanine aminotransferase (ALT), alkaline phosphatase (ALP), gamma glutamyltranspeptidase ( $\gamma$ GT), cholinesterase (ChE), total cholesterol, triglyceride (TG), albumin, fasting plasma glucose (FPG), immuno reactive insulin (IRI), free fatty acids (FFAs), Ferritin, hyaluronic acid, type IV collagen 7S, dehydroepiandrosterone-sulfate (DHEA-S) and insulin-like growth factor-1 (IGF-1). These parameters were measured by the standard techniques used by clinical chemistry laboratories. Body mass index (BMI) was calculated with the following formula: weight in kilograms/ (height in meters) [2]. Obesity was defined as a BMI greater than 25, according to the criteria of the Japan Society for the Study of Obesity. ${ }^{22}$ Patients were assigned a diagnosis of diabetes mellitus
(DM) if a documented use of oral hypoglycemic medication, a random glucose level in excess of $200 \mathrm{mg} / \mathrm{dl}$, or a FPG greater than $126 \mathrm{mg} /$ dl was present. ${ }^{23}$ For diagnosing dyslipidemia, TG and HDL-C were used as parameters. Plasma TG $(>150 \mathrm{mg} / \mathrm{dL})$ and HDL-C $(<40 \mathrm{mg} / \mathrm{dL}$ for males and $<50 \mathrm{mg} / \mathrm{dL}$ for females) as listed in NCEP guidelines. ${ }^{24}$ Hypertension was diagnosed if the patient was on antihypertensive medication and/or had a resting recumbent blood pressure greater than or equal to $140 / 90 \mathrm{mmHg}$ on at least two occasions. The HOMA-IR was calculated on the basis of fasting values of plasma glucose and insulin according to the HOMA model formula: HOMA-IR= IRI $(\mu \mathrm{U} /$ $\mathrm{ml}) \times \mathrm{FPG}(\mathrm{mg} / \mathrm{dl}) \div 405$. Serum concentrations of FT were measured by RIA (the Coat-A-Count: Diagnostic Products Corporation, Los Angeles, CA, USA) ${ }^{25}$ A low level of FT in the serum was defined according to the clinical practice guideline for $\mathrm{LOH}$ of the Japanese Urological Association/Japanese Society for the Study of the Aging Male [11]. According to this guideline, the FT level for diagnosis of $\mathrm{LOH}$ was set at $-2 \mathrm{SD}$ from the mean for men in their $20 \mathrm{~s}(8.5 \mathrm{pg} /$ $\mathrm{mL}$ ). Genotyping for rs738409 (patatin-like phospholipase domaincontaining protein 3: PNPLA3) was accomplished using the TaqMan SNP Genotyping Assay system (Applied Biosystems, Foster City, CA, USA) with the commercially available pre-designed SNP-specific primers for PCR amplification and extension reactions according to the manufacturer's protocol. ${ }^{26}$

\section{Histological evaluation}

Forty-four patients underwent a percutaneous liver biopsy under ultrasonic guidance. The liver specimens were embedded in paraffin and stained with hematoxylin and eosin, Masson-trichrome, and reticulin silver stain. Two hepatopathologists (S.I. and Y.S.) who were blinded to the clinical data reviewed the liver biopsy specimens. An adequate liver biopsy sample was defined as a biopsy specimen with a length greater than $1.5 \mathrm{~cm}$ and/or with more than 6 portal tracts. NASH was defined as steatosis with lobular inflammation and ballooning degeneration with or without the presence of Mallory-Denk bodies or fibrosis. Patients whose liver biopsy specimens showed steatosis, or steatosis with nonspecific inflammation, were identified as the NAFL cohort. ${ }^{2.3}$ The score, named NAS (NAFLD Activity Score) proposed by Kleiner et al. ${ }^{27}$ is the unweighted sum of the scores for steatosis (0-3), lobular inflammation (0-3), and ballooning degeneration (02). ${ }^{27}$ The severity of hepatic fibrosis was defined as follows: Stage 1, zone 3 per sinusoidal fibrosis; Stage 2, zone 3 per sinusoidal fibrosis with portal fibrosis; Stage 3, zone 3 per sinusoidal fibrosis and portal fibrosis with bridging fibrosis; and Stage 4 , cirrhosis. ${ }^{28}$

\section{Statistical analysis}

The results are presented as medians and interquartile ranges for quantitative data or as numbers with percentages in parentheses for qualitative data. Significant differences in quantitative data were determined using the Mann-Whitney $U$ test (Tables 1) (Table $2 \&$ Figure 1). The Fisher's exact probability test or $\chi^{2}$ analysis was used for qualitative data (Tables $1 \& 2$ ). Correlation coefficients were calculated with the Spearman rank correlation analysis. A multivariate analysis was performed by logistic regression analysis to identify variables independently associated with FT levels (Table 3 ) and NASH (Table 4). To assess the accuracy of variables in the differentiation of NASH from NAFL or advanced NAFLD from mild NAFLD, we calculated the sensitivity and the specificity for each value from each test; we then constructed ROC curves by plotting the Se against the reverse $\mathrm{Sp}$ (1 minus $\mathrm{Sp}$ ) for each value (Figure 2). The diagnostic performance of the scoring systems was assessed by analysis of the ROC curves. The most commonly used index of accuracy is the area 
under the ROC curve (AUROC), where values close to 1.0 indicate high diagnostic accuracy. The you den index was used to identify the optimal cut-off points. Significant differences among the three groups with respect to quantitative data were determined by one-way analysis of variance with Scheffe's post hoc test (Figure 3). Differences were considered statistically significant at all Pvalues less than 0.05 .

Table I Characteristics of male patients with NAFLD and control population

\begin{tabular}{|c|c|c|c|}
\hline Parameters & $\begin{array}{l}\text { NAFLD } \\
(n=66)\end{array}$ & $\begin{array}{l}\text { Control } \\
(n=82)\end{array}$ & $\mathbf{P}$ \\
\hline Age $(y r)$ & $57(47,65)$ & $57(49,65)$ & matched \\
\hline BMI $\left(k g / m^{2}\right)$ & $28.0(25.0-29.6)$ & $22.2(20.7,23.7)$ & $<0.001$ \\
\hline Hemoglobin $(g / d L)$ & $15.3(14.5,15.9)$ & $14.5(12.0,15.2)$ & $<0.001$ \\
\hline 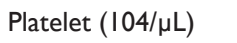 & $21.4(17.0,25.4)$ & $20.8(18.4,24.4)$ & 0.830 \\
\hline AST (IU/L) & $36(25,52)$ & $21(18,24)$ & $<0.00$ I \\
\hline ALT (IU/L) & $56(37,78)$ & $19(15,24)$ & $<0.001$ \\
\hline ALP (IU/L) & $216(191,260)$ & $173(|55,22|)$ & $<0.001$ \\
\hline$\gamma \mathrm{GT}(\mathrm{IU} / \mathrm{I})$ & $47(31,98)$ & $3 I(2 I, 5 I)$ & $<0.001$ \\
\hline Cholesterol (mg/dl) & $199(186,219)$ & $193(|8|, 2 \mid 2)$ & 0.337 \\
\hline Triglyceride (mg/dl) & I54 (I07, 23I) & $103(62,128)$ & $<0.001$ \\
\hline HDL-C (mg/dl) & $46(40,55)$ & $63(51,74)$ & $<0.001$ \\
\hline FPG (mg/dl) & $99(94,108)$ & $94(87,100)$ & 0.014 \\
\hline DHEA-S ( $\mu g / d l)$ & $161(84,2 \mid 3)$ & I $47(\mid 19,208)$ & 0.779 \\
\hline $\mathrm{FT}(\mathrm{pg} / \mathrm{ml})$ & $7.0(5.4,9.2)$ & $9.1(7.2,12.2)$ & $<0.001$ \\
\hline $\mathrm{FT}<8.5 \mathrm{pg} / \mathrm{ml}$ & 45 (68.2\%) & 34 (41.5\%) & 0.002 \\
\hline \multicolumn{4}{|c|}{ Cigarette smoking habits } \\
\hline Never smoker & $34(51.5 \%)$ & $40(48.8 \%)$ & \multirow{3}{*}{0.339} \\
\hline Past smoker & $21(31.8 \%)$ & $18(22.0 \%)$ & \\
\hline Current smoker & II (16.7\%) & 24 (29.3\%) & \\
\hline
\end{tabular}

Results are presented as numbers (\%) for qualitative data or as medians (25\% percentile, 75\% percentile) for quantitative data. BMI: Body Mass Index; AST: Aspartate Aminotransferase; ALT: Alanine Aminotransferase; $\gamma \mathrm{GT}$ : Gamma Glutamyltranspeptidase; HDL-C: High Density Lipoprotein Cholesterol; FPG: Fasting Plasma Glucose; DHEA-S: Dehydroepiandrosterone Sulfate. P-values were calculated by Mann-Whitney $U$ analysis or $\chi^{2}$ analysis.

Table 2 Characteristics of male patients with biopsy-proven NAFLD $(n=44)$

\begin{tabular}{|c|c|c|c|}
\hline Parameters & $\begin{array}{l}\text { NAFL } \\
(n=19[43.2 \%])\end{array}$ & $\begin{array}{l}\text { NASH } \\
(n=25[56.8 \%])\end{array}$ & $\mathbf{P}$ \\
\hline Age (yr) & $50(45,60)$ & $59(45,69)$ & 0.118 \\
\hline BMI $\left(\mathrm{kg} / \mathrm{m}^{2}\right)$ & $28.1(25.7,29.2)$ & $27.9(25.2,30.9)$ & 0.859 \\
\hline Obesity (BMI>25 kg/m²) & I5 (78.9\%) & $20(80.0 \%)$ & 1.000 \\
\hline Diabetes [yes (\%)] & $3(15.8 \%)$ & $7(28.0 \%)$ & 0.474 \\
\hline Hemoglobin (g/dl) & $15.9(15.1,16.3)$ & $15.3(14.6,15.8)$ & 0.106 \\
\hline Platelet $(104 / \mu \mathrm{l})$ & $23.3(19.8,25.8)$ & $19.8(15.3,26.3)$ & 0.222 \\
\hline AST (IU/I) & $34(25,46)$ & $46(35,7 I)$ & 0.050 \\
\hline ALT (IU/I) & $34(58,78)$ & $69(46,103)$ & 0.184 \\
\hline ALP (IU/L) & $217(197,24 I)$ & $230(205,287)$ & 0.250 \\
\hline$\gamma \mathrm{GT}(\mathrm{IU} / \mathrm{I})$ & $39(25,60)$ & $67(45,109)$ & 0.036 \\
\hline Cholinesterase (IU/I) & $421(380,442)$ & $386(329,392)$ & 0.030 \\
\hline Albumin(g/dl) & $4.5(4.4,4.7)$ & $4.5(4.4,4.5)$ & 0.197 \\
\hline Cholesterol (mg/dl) & $198(191,230)$ & $206(190,222)$ & 0.951 \\
\hline Triglyceride (mg/dl) & $149(98,298)$ & $166(1 \mid 1,213)$ & 0.926 \\
\hline HDL-C (mg/dl) & $4 I(35,50)$ & $5 I(43,56)$ & 0.073 \\
\hline
\end{tabular}

Table continued...

\begin{tabular}{|c|c|c|c|}
\hline Parameters & $\begin{array}{l}\text { NAFL } \\
(n=19[43.2 \%])\end{array}$ & $\begin{array}{l}\text { NASH } \\
(n=25[56.8 \%])\end{array}$ & $\mathbf{P}$ \\
\hline Prothrombin time (\%) & $113(106,126)$ & $109(97,114)$ & 0.098 \\
\hline Ferritin $(\mathrm{ng} / \mathrm{ml})$ & $264(228,387)$ & $260(117,309)$ & 0.245 \\
\hline Hyaluronic acid (ng/ml) & $17(11,37)$ & $38(20,72)$ & 0.010 \\
\hline Type IV Collagen 7s (ng/ml) & $4.2(4.1,4.9)$ & $4.9(4.1,6.5)$ & 0.188 \\
\hline FPG $(m g / d l)$ & $98(94.105)$ & $100(94,110)$ & 0.752 \\
\hline IRI $(\mu \mathrm{U} / \mathrm{ml})$ & II.I (7.I, I5.2) & $13.9(11.0,18.1)$ & 0.168 \\
\hline HOMA-IR & $2.7(2.0,3.6)$ & $3.4(2.6,2.9)$ & 0.115 \\
\hline DHEA-S $(\mu g / d l)$ & $212(129,289)$ & $106(67,207)$ & 0.011 \\
\hline IGF-I (ng/ml) & $117(113,159)$ & $108(89,148)$ & 0.343 \\
\hline $\mathrm{FT}(\mathrm{pg} / \mathrm{ml})$ & $8.9(7.2,10.3)$ & $6.0(5.0,7.0)$ & $<0.001$ \\
\hline $\mathrm{FT}<8.5 \mathrm{pg} / \mathrm{ml}$ & $8(42.1 \%)$ & $22(88.0 \%)$ & 0.003 \\
\hline \multicolumn{4}{|l|}{ Cigarette smoking habits } \\
\hline Never Smoker & $8(42.1 \%)$ & $15(60.0 \%)$ & \\
\hline Past Smoker & $6(31.6 \%)$ & $6(24.0 \%)$ & 0.231 \\
\hline Current Smoker & $5(26.3 \%)$ & $4(16.0 \%)$ & \\
\hline $\begin{array}{l}\text { Antidepressant Drugs Use } \\
\text { (yes) }\end{array}$ & I (5.3\%) & I (4.0\%) & 0.595 \\
\hline PNPLA3 SNP (CC/CG/GG) & $\begin{array}{l}3 / 5 / 6 \\
(n=14)\end{array}$ & $\begin{array}{l}2 / 8 / 5 \\
(n=15)\end{array}$ & 0.885 \\
\hline
\end{tabular}

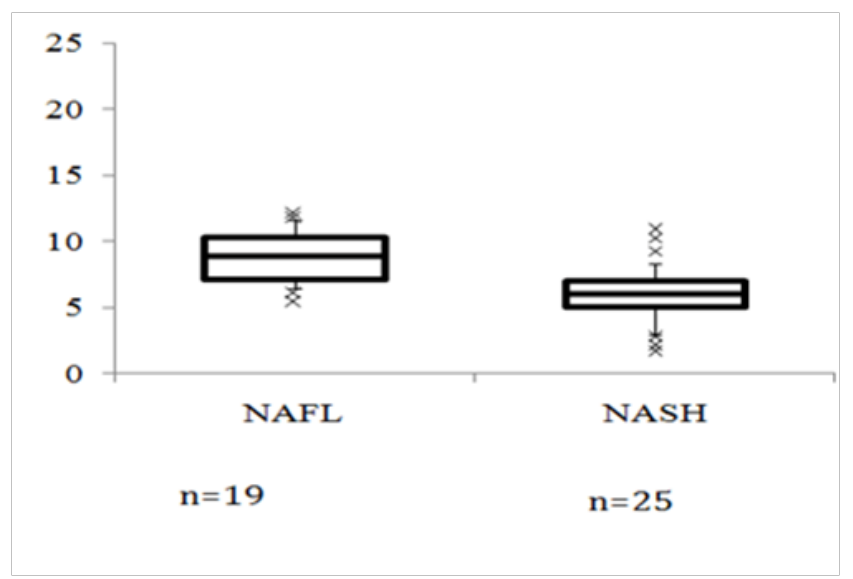

Figure IA Serum free testosterone (FT) levels in patients with NAFLD and in the control group. $\mathrm{FT}(\mathrm{Pg} / \mathrm{ml})$

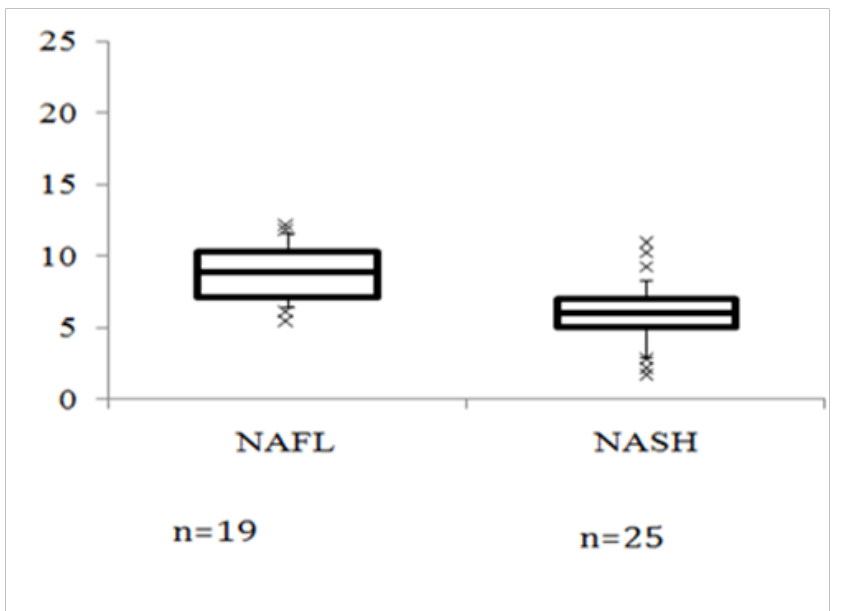

Figure IB Serum FT levels in patients with biopsy-proven NAFL and NASH. 


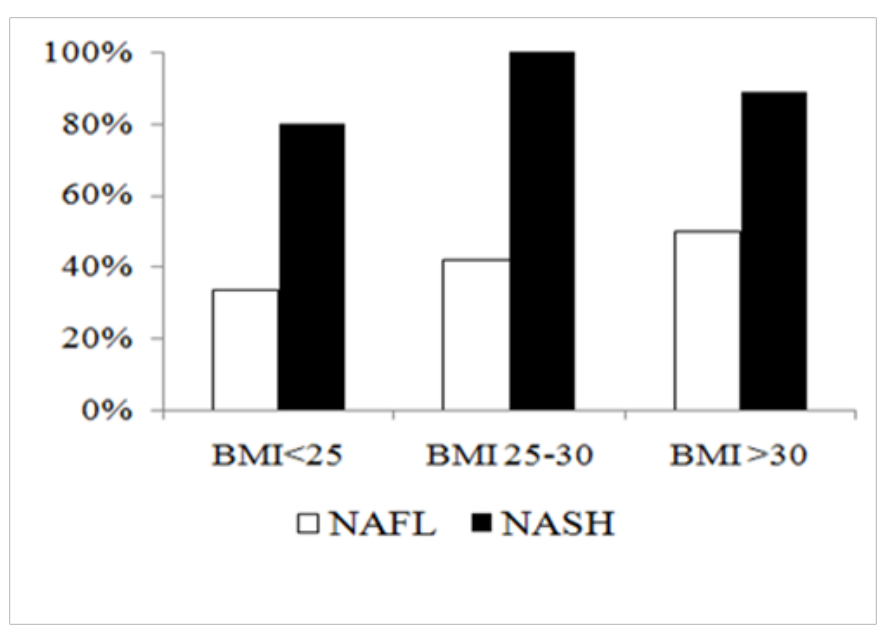

Figure IC The prevalence of low FT levels according to BMI.

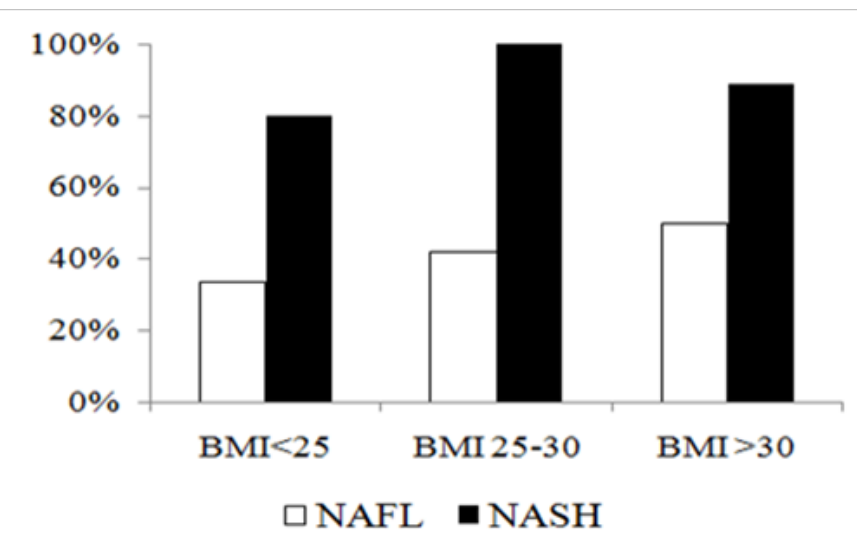

Figure ID The prevalence of low FT levels according to age.

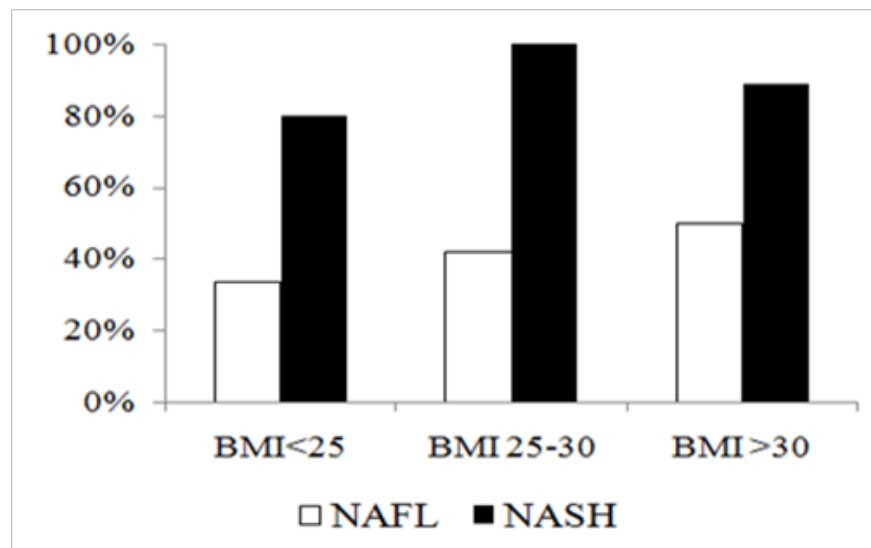

The box comprises the values between the 25 th and 75 th percentiles while the horizontal line indicates the median; the error bars stretch from the 10th to the 90 thpercentile, and individual outliers are represented by crosses. * $\mathrm{P}$ $<0.00$ I vs. control, $* * \mathrm{P}<0.001$ vs. NAFL.

Figure $2 \mathrm{ROC}$ analysis for the differentiation of NASH from NAFL.
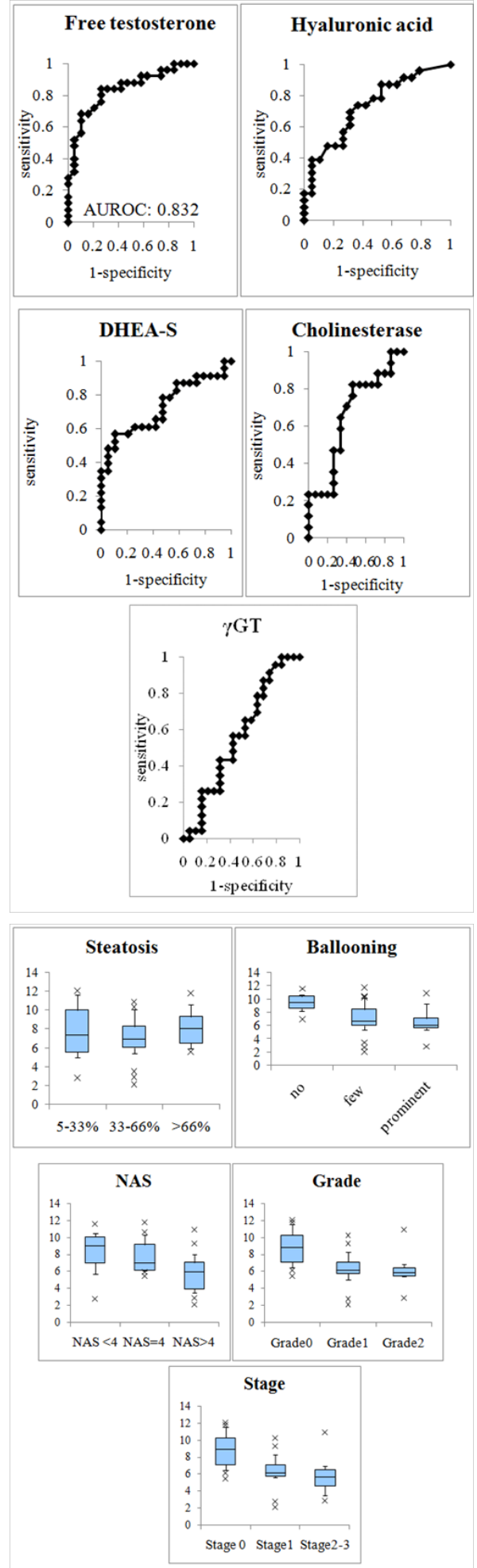

Figure 3 Variation in free testosterone (FT) levels with steatosis, ballooning, NAS, activity, and fibrosis stage for participants with NAFLD.

The box comprises the values between the 25 th and 75 th percentiles while the bold horizontal line indicates the median; the error bars stretch from the 10 th and to the 90 th percentile.

$* P=0.007, * * P=0.028$ vs. Grade $0, \# P=0.007, \# P=0.008$ vs. Stage 0 
Table 3 Multiple regression analysis of correlates with free testosterone (FT)

\begin{tabular}{lllll}
\hline Variables & Standard Partial Regression Coefficient & Standard Error & $\mathbf{9 5 \%} \mathbf{C l}$ & $\mathbf{P}$ \\
\hline Age & -0.017 & 0.032 & $-0.084 \sim 0.049$ & 0.591 \\
ALP & 0.001 & 0.007 & $-0.013 \sim 0.014$ & 0.912 \\
Albumin & 2.690 & 1.379 & $-0.207 \sim 5.588$ & 0.067 \\
Cholinesterase & $\mathbf{0 . 0 1 4}$ & $\mathbf{0 . 0 0 6}$ & $\mathbf{0 . 0 0 0} \mathbf{0 . 0 2 7}$ & $\mathbf{0 . 0 4 8}$ \\
HOMA-IR & $\mathbf{- 0 . 4 0 0}$ & $\mathbf{0 . 1 3 6}$ & $\mathbf{- 0 . 6 8 5} \sim-\mathbf{0 . 1 1 4}$ & $\mathbf{0 . 0 0 9}$ \\
Cholesterol & 0.013 & 0.012 & $-0.012 \sim 0.038$ & 0.298 \\
Prothrombin time & 0.013 & 0.025 & $-0.038 \sim 0.065$ & 0.590 \\
Type IV collagen 7S & -0.149 & 0.364 & $-0.914 \sim 0.616$ & 0.688 \\
DHEA-S & 0.009 & 0.006 & $-0.003 \sim 0.021$ & 0.125 \\
\hline
\end{tabular}

Table 4 Logistic regression models of the association of NASH with FT levels and other clinical variables

\begin{tabular}{|c|c|c|c|}
\hline Variables & OR & $95 \% \mathrm{Cl}$ & $\mathbf{P}$ \\
\hline \multicolumn{4}{|l|}{ Model I } \\
\hline $\mathrm{FT}$ & 0.538 & $0.366-0.791$ & 0.002 \\
\hline \multicolumn{4}{|l|}{ Model 2} \\
\hline FT & 0.559 & $0.378-0.825$ & 0.003 \\
\hline Age & 1.023 & $0.966-1.083$ & 0.437 \\
\hline \multicolumn{4}{|l|}{ Model 3} \\
\hline $\mathrm{FT}$ & 0.522 & $0.348-0.782$ & 0.002 \\
\hline BMI & 1.023 & $0.784-I .123$ & 0.938 \\
\hline \multicolumn{4}{|l|}{ Model 4} \\
\hline $\mathrm{FT}$ & 0.529 & $0.720-0.873$ & 0.013 \\
\hline Age & 1.016 & $0.948-1.089$ & 0.662 \\
\hline BMI & 1.023 & $0.810-1.291$ & 0.849 \\
\hline HOMA-IR & 0.855 & $0.588-1.243$ & $0.41 \mathrm{I}$ \\
\hline \multicolumn{4}{|l|}{ Model 5} \\
\hline FT & 0.530 & $0.320-0.877$ & 0.014 \\
\hline Age & 1.016 & $0.806-1.304$ & 0.658 \\
\hline BMI & 1.025 & $0.810-1.291$ & 0.838 \\
\hline HOMA-IR & 0.853 & $0.584-1.245$ & 0.410 \\
\hline Ferritin & 1.000 & $0.996-1.004$ & 0.934 \\
\hline
\end{tabular}

OR: Odds Ratio; Cl: Confidence Interval; FT: Free Testosterone; BMI: Body Mass Index; HOMA-IR: Homeostasis Model Assessment for Insulin Resistance

\section{Results}

Table 1 summarizes the clinical and laboratory data of the patient population and the control group. Patients with NAFLD were predominantly obese, had higher levels of hemoglobin $(\mathrm{Hb})$, transaminase activity, $\gamma \mathrm{GT}$, TG, and FPG, and had lower levels of HDL-C. The levels of the FT in the serum were significantly lower $(7.0[5.4,9.2] \mathrm{pg} / \mathrm{ml})$ in patients with NAFLD compared to those in the control population $(9.1[7.2,12.2] \mathrm{pg} / \mathrm{ml}, P<0.001$, Figure 1A). Serum levels of FT less than $-2.0 \mathrm{SD}$ from the mean for men in their $20 \mathrm{~s}(8.5 \mathrm{pg} / \mathrm{mL})$ were found in $68.2 \%$ of the patient group compared with $41.5 \%$ of the control group $(P=0.002)$. Of two NAFLD patients who received antidepressant drugs, one patient had paroxetine hydrochloride hydrate (serum FT, 6.4pg/ml), and another had aripiprazole (serum FT, $10.1 \mathrm{pg} / \mathrm{ml}$ ).

In 66 patients with NAFLD, Spearman's rank correlations between FT levels and clinical parameters were calculated. The levels of FT were positively correlated with platelets $(\mathrm{r}=0.290, P=0.030)$, albumin $(\mathrm{r}=0.384, P=0.004), \mathrm{ChE}(\mathrm{r}=0.356, \mathrm{P}=0.028)$, cholesterol $(\mathrm{r}=0.413$, $\mathrm{P}=0.004)$, prothrombin time $(\mathrm{r}=0.281, \mathrm{P}=0.037)$, and DHEA-S $(\mathrm{r}=0.564, \mathrm{P}<0.001)$ and were negatively correlated with age $(\mathrm{r}=-$
$0.288, \mathrm{P}=0.019)$, ALP $(\mathrm{r}=-0.339, \mathrm{P}=0.011)$, IRI $(\mathrm{r}=-0.464, \mathrm{P}=0.005)$, HOMA-IR( $\mathrm{r}=-0.463, \mathrm{P}=0.006)$ and type IV collagen $7 \mathrm{~S}(\mathrm{r}=-0.304$, $\mathrm{P}=0.019)$. The FT level had no association with BMI $(\mathrm{r}=-0.123$, $\mathrm{P}$ $=0.334), \mathrm{Hb}(\mathrm{r}=0.239, \mathrm{P}=0.076)$, AST $(-\mathrm{r}=0.103, \mathrm{P}=0.451)$, ALT $(\mathrm{r}=0.120, \mathrm{P}=0.384), \gamma$ GT $(\mathrm{r}=-0.071, \mathrm{P}=0.604), \mathrm{FPG}(\mathrm{r}=-0.040, \mathrm{P}$ $=0.764)$, TG $(\mathrm{r}=0.065, \mathrm{P}=0.646)$, HDL-C $(\mathrm{r}=-0.052, \mathrm{P}=0.719)$, Ferritin $(\mathrm{r}=0.163, \mathrm{P}=0.222)$, hyaluronic acid $(\mathrm{r}=-0.158, \mathrm{P}=0.242)$, or IGF-1 $(r=0.205, P=0.110)$. To determine the independent determinants of serum FT, we performed a stepwise regression analysis that included variables that were significantly related to serum FT ( $r>0.20$; $\mathrm{P}<0.05$ ). Variables that were independent determinants of serum FT were included in a combination multiple regression analysis (Table $3)$. In this analysis, we found HOMA-IR to be the most important determinant of FT levels $(\mathrm{P}=0.009)$. Another important determinant was the level of $\mathrm{ChE}(\mathrm{P}=0.048)$. Of 44 patients with NAFLD who underwent a liver biopsy, 25 patients $(56.8 \%)$ were histologically diagnosed with NASH. Patients with NASH had lower levels of ChE, and DHEA-S, but had higher levels of GGT, hyaluronic acid, type IV collagen 7S, and HOMA-IR (Table 2). Patients with NASH had lower levels of FT $(6.0[5.0,7.0] \mathrm{pg} / \mathrm{ml})$ than those with NAFL $(8.9$ $[7.2,10.3] \mathrm{pg} / \mathrm{ml}, \mathrm{P}<0.001$ ) (Figure 1B). The percentages of patients 
who demonstrated less than -2.0 SDS of the mean of FT $(8.5 \mathrm{pg} / \mathrm{ml})$ were $42.1 \%$ and $88.0 \%$ in cases of NAFL and NASH, respectively $(P=0.003)$. Diabetic patients presented with similar levels of serum FT $(7.2[6.9,9.1] \mathrm{pg} / \mathrm{ml})$ as non-diabetic patients $(6.4[5.5,9.0] \mathrm{pg} /$ $\mathrm{ml}, \mathrm{P}=0.157)$. Serum FT levels were significantly lower in patients with HOMA-IR $>2.5(6.3[5.7,8.1] \mathrm{pg} / \mathrm{ml})$ and with HOMA-IR $<2.5$ $(8.9[7.3,10.4] \mathrm{pg} / \mathrm{ml}, \mathrm{P}=0.011)$. The prevalence of low FT $(<8.5$ $\mathrm{pg} / \mathrm{ml}$ ) was compared according to histological diagnosis and BMI or according to age in Figure 1C \& 1D. In patients with NAFL, the percentage of low FT increased in proportion to increased BMI or age. Most of the patients with NASH (more than 75\%) exhibited low FT levels, independent of BMI and age. The distribution of the PNPLA genotype was similar between the NAFL and NASH groups. The distribution of smoking habits was similar between the NAFL and NASH groups. Serum FT levels did not differ according to smoking habits (never smoker, $6.1[4.8,9.0] \mathrm{pg} / \mathrm{ml}$; past smoker, $7.3[6.4,8.7]$ $\mathrm{pg} / \mathrm{ml}$; current smoker, $7.0[4.1,9.0] \mathrm{pg} / \mathrm{ml})$.

The AUROC in the separation of patients with and without NASH was the greatest for FT (0.832), followed by hyaluronic acid $(0.733)$, DHEA-S (0.730), cholinesterase (0.659), and $\gamma \mathrm{GT}$ (0.565) (Figure 2). The sensitivity of a FT value of $<7.2 \mathrm{pg} / \mathrm{ml}$ for the presence of NASH was $80.8 \%(21 / 26)$, and the specificity was $73.7 \%(14 / 19)$ The positive predictive value (PPV) of the cutoff value was $80.8 \%$ $(21 / 26)$, and negative predictive value (NPV) was $73.7 \%(14 / 19)$ Logistic regression analysis was performed to find independent variables which are associated with NASH (Table 4). As illustrated in Table 4, the unadjusted (model 1) association of FT levels with the severity of NAFLD remained highly significant even after adjustment for age (model 2) and BMI (model 3). The FT levels remained highly significantly associated with advanced NAFLD after adjustment for insulin resistance or levels of Ferritin in the serum (models 4 and 5).

The association of FT with liver histology was also examined. The levels of FT decreased significantly with increasing lobular inflammation $(\mathrm{P}<0.001)$, ballooning $(\mathrm{P}<0.05)$, NAS $(\mathrm{P}<0.05)$ and fibrosis $(\mathrm{P}<0.001)$. No relationship was observed between the levels of FT and the grade of steatosis (Figure 3). We performed a multiple stepwise regression analysis with FT as the dependent variable and with liver histology parameters (steatosis, ballooning, inflammation, and fibrosis) as independent variables. We found lobular inflammation to be the most important determinant of serum FT levels.

\section{Discussion}

In this study, we first confirmed that serum FT levels are significantly lower in male Japanese patients with NAFLD than in age-matched controls. Then, we found that patients with biopsyproven NASH, a severe form of NAFLD, exhibited significantly lower levels of serum FT compared with those with NAFL even after adjustment for age, BMI, and insulin resistance. The relationship between NAFLD and serum testosterone concentrations has not yet received sufficient attention. A low serum total testosterone level was independently associated with NAFLD in patients in Korea [19] and Germany [20] while serum FT levels were not associated with NAFLD in patients with type2 diabetes [29] and in healthy men [30] in China, and post-menopausal women in Greece. ${ }^{31}$ This discrepancy may be explained by gender distribution (if women were included in the study or not), the diagnostic method of NAFLD (US vs. biopsy), the method of testosterone determination (total testosterone vs. FT), the number of patients involved (small vs. large), and ethnicity (Asian vs. Caucasian). Total testosterone in the serum consists of three components including testosterone bound to sex hormone binding globulin (SHBG), testosterone bound to albumin, and FT. FT and testosterone bound to albumin are called as bioavailable testosterone (BAT). Although FT is a minor component $(2-3 \%)$, it is the only component with hormone activity. In Japan, the Japanese Urological Association and the Japanese Association of Men's Health recommend the measurement of FT levels in the diagnosis of $\mathrm{LOH}$, for the following reasons: 1) It has been demonstrated that FT is more strongly correlated with calculated BAT, than total testosterone, showing a statistically significant difference; 2) The behavior of FT is highly consistent with that of BAT, with FT levels being markedly decreased due to aging; 3 ) For the measurement of FT, a method that allows the measurement of FT only, without affecting the balance between FT and protein-bound testosterone in blood, is available; and 4) The mean total testosterone by age range decreases only to $80 \%$ of the young adult mean even during presenile and senile periods, when LOH occurs most frequently, but the FT level shows a linear decrease with aging and drops to $50 \%$ of the young adult mean. FT is a more accurate parameter of androgen status in conditions of altered SHBG, such as CLD. ${ }^{32}$ We clearly confirmed lower levels of serum FT in Japanese men with NAFLD compared to age-matched healthy people. A higher proportion of patients with NAFLD (68.2\%) presented with serum FT levels below -2 SD of the mean level for men in their $20 \mathrm{~s}(8.5 \mathrm{pg} / \mathrm{mL})^{11}$ compared with the control population $(41.5 \%, P=0.0016)$. This result suggests the association of $\mathrm{LOH}$ syndrome with NAFLD. It is counterintuitive to base the diagnosis of LOH solely on serum FT levels, and it has to be supported by clinical symptoms. ${ }^{11,12}$ Various questionnaires have been constructed over the years to monitor the symptoms of $\mathrm{LOH}$ and their response to testosterone treatment. They include the Aging Male Symptom Score, the Androgen Deficiency in Aging Males. By using these questionnaires, we will examine symptoms, metal status, and quality of life in enrolled patients to realize this association.

The mechanisms that lead to a decrease in serum FT in cases of NAFLD are still unclear. Low serum testosterone levels are closely related with visceral fat accumulation and insulin resistance. ${ }^{33}$ In our study, the serum FT levels were well-correlated with insulin resistance, as evaluated by HOMA-IR. Obesity can decrease the levels of testosterone through the conversion of aromatase to estradiol in adipose tissue and through the release of inflammatory cytokines that can inhibit the hypothalamic-pituitary-gonadal (HPG) axis at multiple levels. According to a recent report by Senmaru et al. ${ }^{34}$ testosterone deficiency increases hepatic TG content via the regulation of the expression of genes involved in the synthesis and secretion of TG and VLDL in the liver such as sterol regulatory element-binding protein 1 , peroxisome proliferator-activated receptor- $\alpha$, and microsomal TG transfer protein. Unfortunately, the expression of these genes was not evaluated in this study. This report is the first to show that patients with NASH had significantly lower levels of FT than patients with NAFL, independent of age, BMI, and insulin resistance. In our multivariate analysis, histological activity was well-correlated with serum FT levels in biopsy-proven NAFLD. Testosterone has been known to have a variety of functions, including anti-inflammatory properties and anti-oxidative effects. First of all, low serum testosterone is associated with markers of inflammation. ${ }^{35,36}$ Testosterone was found to attenuate inflammatory cytokines ${ }^{37}$ and to up-regulate antiinflammatory cytokine. ${ }^{38}$ Significant improvement has been shown in the levels of inflammatory markers following androgen replacement therapy in hypogonadal men with metabolic syndrome. ${ }^{39,40}$ On the other hand, locally produced inflammatory cytokines such as TNF $\alpha$, interleukin 1(IL-1), and IL-6can markedly stimulate the aromatase activity in peripheral tissues with subsequent increased conversion 
of testosterone to estradiol. These cytokines are reported to play an important role in the development of NAFLD, although inflammatory cytokines were not measured in this study. There appears to be a bidirectional relationship between decreased levels of serum FT and hepatic inflammation. Oxidative stress could be another possible link between low levels of testosterone and NASH, as oxidative stress may be important in the pathogenesis of NASH.8,9Accumulating evidence shows that testosterone exhibits antioxidant effects, although the precise mechanisms remain unknown. ${ }^{41,43}$ Taken into consideration, it is plausible that low levels of testosterone can exacerbate hepatic inflammation or oxidative stress which might lead to NASH or fibrosis.

Currently, liver biopsy is the gold standard for the diagnosis of $\mathrm{NASH}$, and no established parameters exist for the prediction of NASH without a liver biopsy. ${ }^{44}$ ROC analysis exhibited the greatest AUROC for FT among several parameters which demonstrated a significant difference between NAFL and NASH. This result also suggests that determinations of serum FT may help us distinguish NASH from NAFL in male patients with NAFLD. A "dose effect" of lower FT with advanced fibrosis was observed with a median level of FT of $8.9,6.2$, and $5.7 \mathrm{pg} / \mathrm{ml}$ for fibrosis stages 0,1 , and $2-3$, respectively. The mechanisms that underlie the association between decreased FT and advanced fibrosis are still largely unknown. HPG dysfunction in patients with cirrhosis was discovered more than three decades ago. Irrespective of etiology, patients with cirrhosis had significantly reduced concentrations of FT. ${ }^{45}$ Men with chronic non-alcoholic liver disease had reduced levels of FT, which were correlated with disease severity and were improved after hepatic transplantation. ${ }^{46} \mathrm{~A}$ recent study demonstrated the protective effects of testosterone on the pathogenesis of hepatic steatosis in rats that were fed a high-fat diet. ${ }^{47}$ In 117 hypogonadal elderly men, the levels of ALT, AST, and CRP had decreased significantly after one year of testosterone treatment. ${ }^{48}$ In that study, a remarkable improvement in body weight, BMI and waist circumference was observed along with an improvement in lipid profiles. We are now performing a pilot study to investigate whether testosterone administration can improve NASH histology as well as the results of liver function tests in the future. On the other hand, it is possible that caloric restriction can increase serum testosterone concentrations in obese male subjects. ${ }^{49}$ In the near future, we should investigate serial changes of serum FT levels after body weight reduction or medical treatments for NASH/NAFLD.

Our study has several important limitations. Unfortunately, we did not assess the associations of serum FT with erectile dysfunction, atherosclerosis, or sarcopenia. These associations should be clarified in NAFLD patients. Unfortunately, we could not examine SHBG, protein-bound testosterone and total testosterone because of high cost and a small amount of frozen sera. There may have been patient selection bias because a liver biopsy might be considered for patients with NAFLD who are likely to have NASH. We acknowledge that the pathological diagnosis was primarily determined with liver tissues that were obtained by percutaneous liver biopsy, which is prone to sampling error or inter-observer variability. Since all subjects in the present study were Japanese men, the present results cannot simply be extrapolated to other ethnic groups. Further longitudinal cohort studies are needed to verify the causal relationship between NAFLD and FT levels, and well-designed randomized controlled trials are necessary to determine whether testosterone replacement therapy can improve NAFLD/NASH. Despite these limitations, a significant contribution of this study is that it is the first one to identify the association of FT with NASH. In conclusion, we have found that patients with NAFLD, especially with NASH, have low circulating levels of FT. These data provide novel evidence for FT deficiency in male Japanese patients with histologically advanced NASH, independent of age, obesity, and insulin resistance.

\section{Acknowledgement}

The authors thank the following individuals for assistance in preparation of this manuscript: Hitoshi Toda, M.D. Oike Clinic, and Kayo Endo, Nara City Hospital.

\section{Conflicts of interest}

The authors have no conflict of interest for this research.

\section{References}

1. Watanabe S, Hashimoto E, Ikejima K, et al. Evidence-based clinical practice guidelines for nonalcoholic fatty liver disease/nonalcoholic steatohepatitis. J Gastroenterol. 2015;50(4):364-377.

2. Matteoni CA, Younossi ZM, Gramlich T, et al. Nonalcoholic fatty liver diseases: a spectrum of clinical and pathological severity. Gastroenterology . 1999;116(6):1413-1419.

3. Rafiq N, Fang Y, McCullough A, et al. Long-term follow-up of patients with nonalcoholic fatty liver. Clin Gastroenterol Hepatol. 2009; 7(2):234-238.

4. Nishioji K, Sumida Y, Kamaguchi M, et al. Prevalence of and risk factors for non-alcoholic fatty liver disease in anon-obese Japanese population, 2011-2012. J Gastroenterol. 2015;50(1):95-108.

5. Saibara T. Nonalcoholic steatohepatitis in Asia-Oceania. Hepatol Res. 2005;33(2): 64-67.

6. Kessoku T, Yoneda M, Sumida Y, et al. Insulin resistance correlated with the severity of liver histology in Japanese NAFLD patients: a multicenter retrospective study. J Clin Gastroenterol. 2015;49(1):169-170.

7. Sumida Y, Nakashima T, Yoh T, et al. Serum thioredoxin levels as a predictor of steatohepatitis in patients with nonalcoholic liver disease. $J$ Hepatol. 2003;38(1):32-38.

8. Sumida Y, Niki E, Naito Y, et al. Involvement of free radicals and oxidative stress in NAFLD/NASH. Free Radic Res. 2013;47(11): 869880 .

9. Sumida Y, Yonei Y, Tanaka S, et al. Lower levels of insulin-like growth factor -1 standard deviation score are associated with histological severity of non-alcoholic fatty liver disease. Hepatol Res. 2015;45(7):771-781.

10. Okamura K, Ando F, Shimokata H. Serum total and free testosterone level of Japanese men: a population-based study. Int $J$ Urol. 2005;12(9):810-814.

11. Namiki M, Akaza H, Shimazui T, Ito N, et al. Clinical practice manual for late-onset hypogonadism syndrome. Int J Urol. 2008;15(5): 377388 .

12. Iwamoto T, Yanase T, Horie H, et al. Late-onset hypogonadism (LOH) and androgens: validity of the measurement of free testosterone levels in the diagnostic criteria in Japan. Int J Urol. 2009;16(2):168-674.

13. Katabami T, Kato H, Asahina T, et al. Serum free testosterone and metabolic syndrome in Japanese men. Endocr J. 2010;57(6):533-539.

14. Fukui M, Soh J, Tanaka M, et al. Low serum testosterone concentration in middle-aged men with type 2 diabetes. Endocr J. 2007;54(6):871877.

15. Fukui M, Kitagawa Y, Nakamura N, et al. Association between serum testosterone concentration and carotid atherosclerosis in men with type 2 diabetes. Diabetes Care. 2003;26(6):1869-1873. 
16. Tsujimura A, Yamamoto R, Okuda H, et al. Low serum free testosterone level is associated with carotid intima-media thickness in middle-aged Japanese men. Endocr J. 2012;59(9):809-815.

17. Imai A, Yamamoto $\mathrm{H}$, Hatakeyama $\mathrm{S}$, et al. Risk factors for erectile dysfunction in healthy Japanese men. Int J Androl. 2010;33(4): 569-573.

18. Yuki A, Otsuka R, Kozakai R, et al. Relationship between low free testosterone levels and loss of muscle mass. Sci Rep. 2013:1818.

19. Kim S, Kwon H, Park JH, et al. A low level of serum total testosterone is independently associated with nonalcoholic fatty liver disease. BMC Gastroenterol. 2012;12:69.

20. Völzke H, Aumann N, Krebs A, et al. Hepatic steatosis is associated with low serum testosterone and high serum DHEAS levels in men. Int $J$ Androl. 2013;33(1):45-53.

21. Tanaka K, Hyogo H, Ono M, et al. Upper limit of normal serum alanine aminotransferase levels in Japanese subjects. Hepatol Res. 2014;44(12):1196-1207.

22. Japanese Society for the Study of Obesity. New criteria of obesity (in Japanese). J Jpn Soc Study Obes. 2000;6:18-28.

23. American Diabetes Association. Report of the expert committee on the diagnosis and classification of diabetes mellitus. Diabetes Care. 1997;20:1183-1197.

24. National Cholesterol Education Program (NCEP) Expert Panel on Detection. Evaluation and treatment of high blood cholesterol in adults (Adult Treatment Panel III) executive summary of the third report. JAMA. 2001; 285: 2486e97.

25. Yanase T, Haji M, Natori S, et al. Fundamental and clinical studies on a solid-phase radioimmunoassay kit for the quantitative measurement of free testosterone in serum. Clinical Endocrinology (Tokoyo). 1998;69:357-362.

26. Romeo S, Kozlitina J, Xing C, et al. Genetic variation in PNPLA3 confers susceptibility to nonalcoholic fatty liver disease. Nat Genet. 2008;40(12):1461-1465.

27. Kleiner DE, Brunt EM, Van Natta M, et al. Design and validation of a histological scoring system for nonalcoholic fatty liver disease. Hepatology. 2005;41(6):1313-1321.

28. Brunt EM, Janney CG, Di Bisceglie AM, et al. Non-alcoholic steatohepatitis: a proposal for grading and staging the histological lesions. Am. J. Gastroenterol. 1999;94(9):2467-2474.

29. Hua X, Sun Y, Zhong Y, et al. Low serum sex hormone--binding globulin is associated with nonalcoholic fatty liver disease in type 2 diabetic patients. Clin Endocrinol (Oxf). 2014;80(6):877-883

30. Tian GX, Sun Y, Pang CJ, et al. Oestradiol is a protective factor for nonalcoholic fatty liver disease in healthy men. Obes Rev. 2014;13(4):381387.

31. Polyzos SA, Kountouras J, Tsatsoulis A, et al. Sex steroids and sex hormone-binding globulin in postmenopausal women with nonalcoholic fatty liver disease. Hormones (Athens). 2013;12(3):405-416.

32. Sinclair M, Grossmann M, Gow PJ, et al. Testosterone in men with advanced liver disease: abnormalities and implications. J Gastroenterol Hepatol. 2015;30(22): 2441-251

33. Fui MN, Dupuis P, Grossmann M. Lowered testosterone in male obesity: mechanisms, morbidity and management. Asian J Androl. 2014;16(2): $223-231$
34. Senmaru T, Fukui M, Okada H, et al. Testosterone deficiency induces markedly decreased serum triglycerides, increased small dense LDL, and hepatic steatosis mediated by dysregulation of lipid assembly and secretion in mice fed a high-fat diet. Metabolism. 2013;62(6): 851-860.

35. Bobjer J, Katrinaki M, Tsatsanis C, et al. Negative association between testosterone concentration and inflammatory markers in young men: a nested cross-sectional study. PLoS One. 2013;8(4): e61466.

36. Tsilidis KK, Rohrmann S, McGlynn K, et al. Association between endogenous sex steroid hormones and inflammatory biomarkers in US men. Andrology. 2013;1(6): 919-928.

37. Li ZG, Danis VA, Brooks PM. Effect of gonadal steroids on the production of IL-1 and IL-6 by blood mononuclear cells in vitro. Clin Exp Rheumatol. 1993;11(2):157-162.

38. Liva SM, Voskuhl RR. Testosterone acts directly on CD4+ T lymphocytes to increase IL-10 production. J Immunol. 2001;167(4): 2060-2067.

39. Malkin CJ, Pugh PJ, Jones RD, et al. The effect of testosterone replacement on endogenous inflammatory cytokines and lipid profiles in hypogonadal men. J Clin Endocrinol Metab. 2004;89(7):3313-3318.

40. Kalinchenko SY, Tishova YA, Mskhalaya GJ, et al. Effects of testosterone supplementation on markers of the metabolic syndrome and inflammation in hypogonadal men with the metabolic syndrome: the double-blinded placebo-controlled Moscow study. Clin Endocrinol (Oxf). 2010;73(5): 602-612.

41. Mancini A, Leone E, Festa R, et al. Effects of testosterone on antioxidant systems in male secondary hypogonadism. J Androl. 2008;29(6):622629

42. Fanaei H, Karimian SM, Sadeghipour HR, et al. Testosterone enhances functional recovery after stroke through promotion of antioxidant defenses, BDNF levels and neurogenesis in male rats. Brain Re. 2014;1558:74-83.

43. Zhang L, Wu S, Ruan Y, et al.Testosterone suppresses oxidative stress via androgen receptor-independent pathway in murine cardiomyocytes. Mol Med Rep. 2011;4(6):1183-1188.

44. Sumida Y, Nakajima A, Itoh Y. Limitations of liver biopsy and noninvasive diagnostic tests for the diagnosis of nonalcoholic fatty liver disease/nonalcoholic steatohepatitis. World $J$ Gastroenterol. 2014;20(2):475-485.

45. Mowat NA, Edwards CR, Fisher R, et al. Hypothalamic-pituitarygonadal function in men with cirrhosis of the liver. Gut. 1976;17(5):345350 .

46. Handelsman DJ, Strasser S, McDonald JA, et al. Hypothalamicpituitary-testicular function in end-stage non-alcoholic liver disease before and after liver transplantation. Clin Endocrinol (Oxf). 1995;43(3):331-337.

47. Nikolaenko L, Jia Y, Wang C, et al. Testosterone replacement ameliorates nonalcoholic fatty liver disease in castrated male rats. Endocrinology. 2014;155(2):417-428.

48. Haider A, Gooren LJ, Padungtod P, et al. Improvement of the metabolic syndrome and of non-alcoholic liver steatosis upon treatment of hypogonadal elderly men with parenteral testosterone undecanoate. Exp Clin Endocrinol Diabetes. 2010;118(3):167-671.

49. Schulte DM, Hahn M, Oberhäuser F, et al. Caloric restriction increases serum testosterone concentrations in obese malesubjects by two distinct mechanisms. Horm Metab Res. 2014;46(4):283-286. 